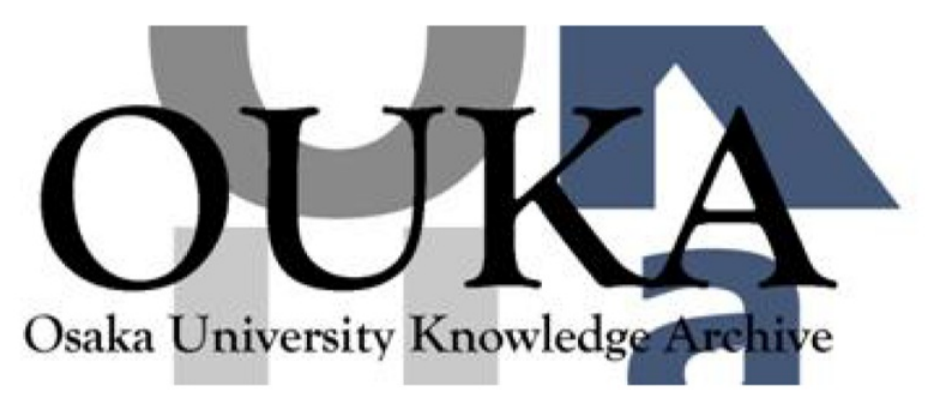

\begin{tabular}{|c|l|}
\hline Title & $\begin{array}{l}\text { Rapid breakdown mechanisms of open air } \\
\text { nanosecond dielectric barrier discharges }\end{array}$ \\
\hline Author(s) & $\begin{array}{l}\text { Ito, Tsuyohito; Kanazawa, Tatsuya; Hamaguchi, } \\
\text { Satoshi }\end{array}$ \\
\hline Citation & Physical Review Letters. 107(6) p. 065002 \\
\hline Issue Date & $2011-08-03$ \\
\hline oaire:version & VoR \\
\hline URL & https://hdl. handle.net/11094/78473 \\
\hline rights & $\begin{array}{l}\text { Copyright (2011) by the American Physical } \\
\text { Society. }\end{array}$ \\
\hline Note & \\
\hline
\end{tabular}

Osaka University Knowledge Archive : OUKA

https://ir. Library. osaka-u. ac. jp/

Osaka University 


\title{
Rapid Breakdown Mechanisms of Open Air Nanosecond Dielectric Barrier Discharges
}

\author{
Tsuyohito Ito, ${ }^{1,2, *}$ Tatsuya Kanazawa, ${ }^{2}$ and Satoshi Hamaguchi ${ }^{2}$ \\ ${ }^{1}$ Frontier Research Base for Global Young Researchers, Graduate School of Engineering, Osaka University, Osaka 565-0871, Japan \\ ${ }^{2}$ Center for Atomic and Molecular Technologies, Graduate School of Engineering, Osaka University, Osaka 565-0871, Japan
}

(Received 18 April 2011; published 3 August 2011)

\begin{abstract}
The discharge initiation mechanism of nanosecond dielectric barrier discharges in open air has been clarified with time-dependent measurement of the discharge electric field by electric-field-induced coherent Raman scattering and optical emission. Our experimental observations have revealed that, in the prebreakdown phase of a nanosecond dielectric barrier discharge, the externally applied fast-rising electric field is strongly enhanced near the cathode due to large accumulation of space charge, which then strongly enhances ionization near the cathode. Once a sufficiently large number of ionizations take place, the location of peak ionization forms a front and propagates toward the cathode with strong optical emission, which establishes the discharge. This process is essentially different from the well-known Townsend mechanism for slower discharges.
\end{abstract}

DOI: 10.1103/PhysRevLett.107.065002

PACS numbers: 52.80.- s, 42.65.Dr, 52.25.Jm

Atmospheric pressure plasmas with low gas temperature, especially those made by dielectric barrier discharges (DBDs), are used in various applications [1-5]. A better understanding of the discharge mechanisms of such plasmas is expected to contribute to the development of better plasma-generation systems specific for each application. When atmospheric pressure plasmas are used with low gas temperature, plasmas are typically generated as a series of pulsed discharges so that the ambient gas is cooled sufficiently after each discharge pulse. Each discharge pulse can last only for a very short period (e.g., on the order of a few dozen nanoseconds), and therefore the discharge initiation process often characterizes the nature of such a discharge pulse. However, detailed discharge characteristics in the initiation phases of atmospheric pressure plasmas are far from being well understood, mostly because only a few diagnostic techniques are available for the measurement of such plasmas.

For a parallel plate discharge, if the electric field generated between the electrodes evolves slowly to the extent that the field may be considered to be nearly stationary (i.e., constant in time and uniform in space) with respect to electron and ion motions, an electron avalanche takes place between the electrodes initiated by a small number of preexisting electrons when the field is sufficiently strong. According to the Townsend theory [6], if ions generated in the electron avalanche are transported to the cathode and generate sufficiently many secondary electrons there, then the electron avalanche continues or even grows in time. When this cycle of secondary electron emission by ion impact and ion generation by secondary electrons is established, "breakdown" is considered to have taken place. Prior to breakdown (i.e., in the prebreakdown phase), the externally applied field essentially remains uniform in space, and, after breakdown, the field develops its spatial variation due to accumulated space charges. This rapid evolution of the electric field typically manifests itself as cathode-directed ionization front propagation [7]. What is essential for discharge breakdown in the Townsend theory is incessant emission of secondary electrons. In other words, in the Townsend theory, breakdown would not occur unless ions move across the electrode gap to generate secondary electrons $[8,9]$.

However, if the externally applied electric field evolves far more rapidly than ions cross the electrode gap, a totally different discharge breakdown process can take place. In such a process, a discharge breakdown typically takes place before most ions in the system have enough time to reach the cathode [10]. This does not mean that secondary electrons play unimportant roles in such a discharge, but obviously a cycle of secondary electron emission by ion impact and ion generation by secondary electrons, as in the Townsend theory, does not explain the discharge breakdown. If secondary electron emission of the Townsend theory is not essential for breakdown, what is the breakdown mechanism? The goal of this study is to answer this question by observing carefully the initiation phase of DBDs in air. The observations we made for this study are the time evolutions of electric fields and optical emission in the gap of parallel plate discharge electrodes.

The experiments were conducted with a parallel plate DBD discharge system. Each electrode is made of brass and is circular in shape with a diameter of $6 \mathrm{~mm}$. It is covered with a glass plate of $0.15 \mathrm{~mm}$ in thickness whose relative permittivity is about 4.6. The gap distance, defined here as the distance between the dielectric surfaces, is $0.85 \mathrm{~mm}$ with the accuracy of about $0.05 \mathrm{~mm}$. With this configuration, the discharge is essentially one-dimensional in space. During the measurements presented in this Letter, the temperature and the humidity of the environment were approximately $300 \mathrm{~K}$ and $50 \%$, respectively. 


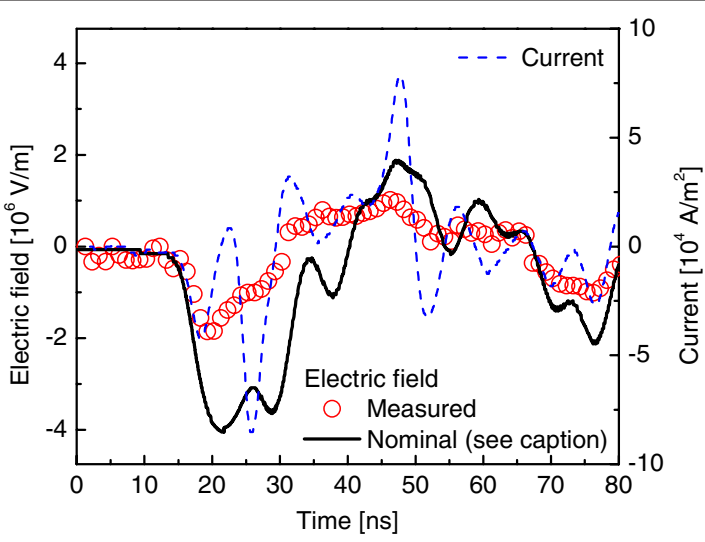

FIG. 1 (color online). Temporal evolution of the nominal electric field (the electric field expected without any space charge: solid curve), electric field at the center of the gap measured by E-CRS (empty circle), and the current (dashed curve).

The repetitively pulsed nanosecond voltage was applied to one of the electrodes (which will be referred to as the "cathode" in this Letter) by a power supply (FID GmbH: FPG20-100MC2). The other electrode is connected to the ground. The peak voltage was about $-3.5 \mathrm{kV}$, and its duration with discharge generation was about $15 \mathrm{~ns}$ in FWHM, with the repetition rate of $10 \mathrm{kHz}$. The applied voltage is proportional to the "nominal electric field," which is defined here as $93 \%$ of the applied voltage divided by the gap length and shown by a solid curve in Fig. 1. (7\% of the applied voltage is taken up by the dielectric barrier layers on the electrodes.) The discharge current measured with a current probe (Pearson Electronics: 6585) at the ground electrode is also shown by the broken curve in Fig. 1. The gas temperature estimated from the coherent anti-Stokes Raman scattering signal reduction throughout the experiments of this study is at most $340 \mathrm{~K}$.

The electric field was measured by electric-fieldinduced coherent Raman scattering (E-CRS) [10-14]. The principle of the electric-field measurement by E-CRS with nitrogen has been demonstrated recently [14]. However, the study in this Letter represents the first measurement of this kind in air plasmas. A schematic diagram of the discharge system is shown in Fig. 2. The setup of the E-CRS measurement system is the same as that for hydrogen discharges, reported in Ref. [10], except for the wavelength and the detector arrangements for Raman signals from nitrogen molecules, which are reported in Ref. [14]. Other parameters are the following. Two pulsed nanosecond laser beams of wavelengths of 532 (at $10 \mathrm{~mJ}$ ) and $607 \mathrm{~nm}$ (at $5 \mathrm{~mJ}$ ) with pulse durations of 3-5 ns in FWHM are employed. For a scientific measurement, the averaged value over 50 signals is used. As to system calibration (for sensitivity and relative timing between laser and power supply), we performed a time-dependent field measurement in the absence of a discharge as in the previous study [10], taking into account the fact that $93 \%$ and $7 \%$ of the applied voltage appear in the gap and

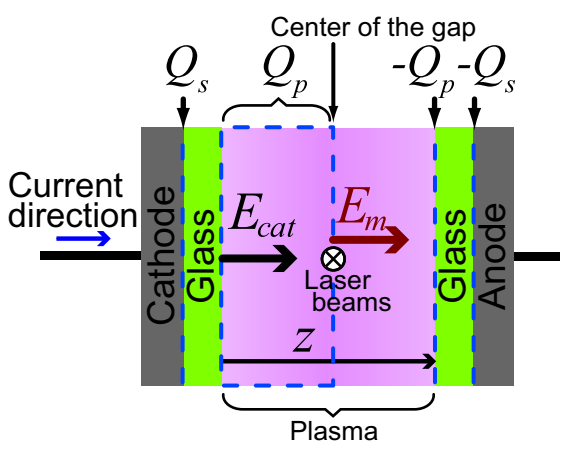

FIG. 2 (color online). A schematic diagram of the discharge system. The arrows illustrate the sign convention of current and electric-field directions employed in this study. The electric field was measured by E-CRS at the center of the electrode gap, and its value is denoted by $E_{m}$. The electric field at the cathode is denoted by $E_{\text {cat }}$.

dielectrics, respectively. Time evolution of the electric field measured at the center of the gap is shown by open circles in Fig. 1. It is clearly seen that the measured electric field deviates from the nominal electric-field value at around 19 ns, which indicates the presence of space charge.

Figure 3 shows the emission intensity as a function of the time and position measured by an intensified charged coupled device camera (Hamamatsu Photonics: C848405G01 and C7164-03). Its gate width is $250 \mathrm{ps,} \mathrm{and} \mathrm{the}$ time step is 100 ps. The images are averaged over 1000 samples. No filter was employed, and therefore all the visible light from the discharge has been captured. The horizontal axis for time is essentially the same as that for Fig. 1. Synchronization of the time axis between Figs. 1 and 3 was achieved by equating the time for the lowest electric-field strength of Fig. 1 with the time for the weakest optical emission of Fig. 3 (at around $30.5 \mathrm{~ns}$ ). The vertical axis represents the position measured from the dielectric surface of the cathode.

Figure 3(a) shows that strong optical emissions appear at around 23 and $47 \mathrm{~ns}$. Relatively weak emissions are also seen at around 26, 36, 41, and $76 \mathrm{~ns}$. The expanded view of the emission at around $23 \mathrm{~ns}$ is given in Fig. 3(b), where an optical emission is seen to propagate from a region near the anode towards the cathode with a velocity of $10^{6} \mathrm{~m} / \mathrm{s}$. It should be noted that, at $19 \mathrm{~ns}$ when the electric field clearly deviates from the nominal field in Fig. 1, no emission is seen in Fig. 3. This indicates that a significant space charge accumulates well before the discharge breakdown (accompanied by strong optical emission).

If we denote the electric field measured at the center $(z=0.425 \mathrm{~mm}$, with $z$ being the distance from the dielectric surface of the cathode) as $E_{m}$ and the charge on the cathode metal per unit area of the electrode surface as $Q_{s}$, then the space charge $Q_{p}$ between $z=0$ and $z=0.425 \mathrm{~mm}$ per unit area of the discharge cross section (i.e., the plane parallel to the electrode surface) is given by $Q_{p}=\varepsilon_{0} E_{m}-Q_{s}$ from Gauss's law. The cathode charge 

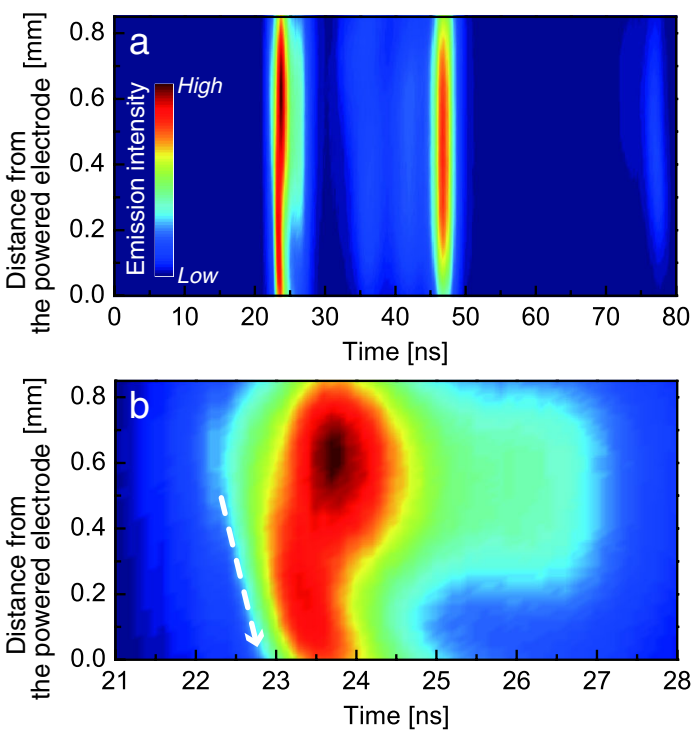

FIG. 3 (color online). Spatiotemporal distribution of the emission intensity obtained from an intensified charged coupled device camera; (a) 0-80 ns, (b) 21-28 ns. The white dashed arrow in (b) is a guide to the eye for the emission propagation with a propagation velocity of approximately $10^{6} \mathrm{~m} / \mathrm{s}$.

$Q_{s}$ is evaluated from the integration of the cathode current shown in Fig. 1, assuming that the dielectric glass completely blocks the current. Time evolution of the estimated charge $Q_{p}$ in the half-space near the cathode is shown in Fig. 4. For reference, emission intensities given in Fig. 3(a) are also superimposed on Fig. 4. Clear synchronization is seen between the optical emission and charge $Q_{p}$, such as strong optical emission at the inflection point of charge evolution at around $23 \mathrm{~ns}$, the lowest emission near the charge maximum at around $30 \mathrm{~ns}$, and another strong emission near the change in charge polarity at around $46 \mathrm{~ns}$, which indirectly supports the assumption employed for time synchronization between Figs. 1 and 3 mentioned earlier. In this Letter, we focus on space charge effects in the discharge initiation phase. Discussion on a later phase will be presented elsewhere.

It is interesting to note that a significantly large net charge $\left(Q_{p} \approx 1.5 \times 10^{-4} \mathrm{C} / \mathrm{m}^{2}\right)$ is already accumulated in the half-space near the cathode prior to detectable optical emission (breakdown) at around $23 \mathrm{~ns}$. Since the maximum charge there during the discharge is approximately $4 \times 10^{-4} \mathrm{C} / \mathrm{m}^{2}$, as seen at around $30 \mathrm{~ns}$ in Fig. 4, more than one-third of the maximum net charge is shown to be formed already in the prebreakdown phase. The positive charge shown here is likely to come from a tenuous plasma that is a remnant from the preceding discharge pulse. When a large electric field is applied, the electrons move towards the anode, leaving behind the positive ions, which hardly move in the time scale considered here. While many electrons may be attached to the dielectric surface of the anode (i.e., grounded electrode in our system), most ions stay in space. Assuming that the

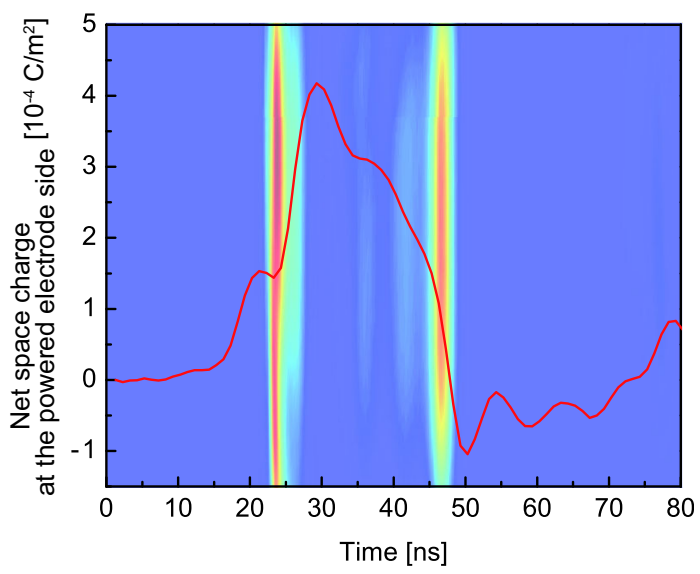

FIG. 4 (color online). The estimated charge $Q_{p}$ in the halfspace near the cathode, evaluated from the electric field and charge on the cathode. The background is the map of optical emissions of Fig. 3 with slightly lighter color tones.

electron motion is mostly of electric-field drift, its velocity in the $z$ direction is given by $v=\mu E$, with $\mu$ and $E$ being the electron mobility and electric field, respectively. The distance $d_{e}$ of electron drift between time $t=0$ and $T$ is then given by

$$
d_{e}=\left|\mu \int_{0}^{T} E d t\right|
$$

which is about $0.3 \mathrm{~mm}$ at $T=18 \mathrm{~ns}$ with $\mu=5.9 \times 10^{-2} \mathrm{~m}^{2} \cdot \mathrm{V}^{-1} \cdot \mathrm{s}^{-1}[6]$ and the integral of the electric field being $-5 \times 10^{-3} \mathrm{~V} \cdot \mathrm{m}^{-1} \cdot \mathrm{s}$ evaluated from the field values at the center given in Fig. 1. If the initial plasma is uniformly distributed, then the net positive charge $Q_{p}$ at $T=18 \mathrm{~ns}$ may be given from the relation $Q_{p}=e d_{e} n_{i 0}$, with $e$ and $n_{i 0}$ being the elementary charge and ion density of the initial plasma, respectively. Using the values given above and Fig. 4, we obtain $n_{i 0} \approx 2 \times 10^{18} \mathrm{~m}^{-3}$.

In the prebreakdown phase, space charge developed in this phase shields the electric field. In an attempt to maintain a high voltage between the electrodes, the power supply pumps more electrons to the cathodes, resulting in a larger electric field at and near the cathode. Using $Q_{s}$ obtained from the integration of current given in Fig. 1, we evaluate the electric field at the cathode $E_{\text {cat }}$ from the relation $E_{\text {cat }}=Q_{s} / \varepsilon_{0}$, assuming that few ions have been adsorbed by the cathode dielectric surface as there is little time for most ions reach the surface. For example, at $t=19 \mathrm{~ns}$, the cathode charge per unit area is approximately $-10^{-4} \mathrm{C} / \mathrm{m}^{2}$, which means $E_{\text {cat }} \approx-10^{7} \mathrm{~V} / \mathrm{m}$, significantly larger in magnitude than the nominal electric field given in Fig. 1. This is an enhancement of the electric field near the cathode in the prebreakdown phase due to charge accumulation.

Since the electron drift motion of preexisting plasma (i.e., a remnant from the preceding discharge pulse) toward 
the anode that leaves behind nearly motionless ions is the first step for the enhancement of the electric field near the cathode, such electrons have little chance to be accelerated by the enhanced field. On the other hand, secondary electrons are the ones that are accelerated by the electric field near the cathode. Although ions hardly move in the time scale of interest, ions that are initially located very close to the cathode do reach the cathode in this time scale and generate some secondary electrons. The number of secondary electrons may be small. However, their acceleration by the enhanced electric field and consequently their ability to ionize may not be neglected, since the ionization coefficient $\alpha$ has a strong dependence on the electric field [15]. Once a sufficiently large number of ionizations take place, the location of peak ionization forms a front and propagates toward the cathode with strong optical emission, which establishes the discharge breakdown.

In summary, from time-resolved experimental measurements of fast initiation phases of parallel plate DBDs, we have shown that, when the externally applied field evolves much faster than the time scale for ions to move across the gap, the discharge breakdown is caused by a mechanism essentially different from Townsend theory. With a fast evolving externally applied field, a discharge breakdown takes place well before ions generated by ionization in the bulk impact the cathode and cause secondary electron emission. In the prebreakdown phase of such a discharge, significant charge accumulation occurs. This charge accumulation is caused by charge separation of the initial plasma remaining from the previous discharge pulse as well as ionization caused by secondary electrons accelerated by the enhanced field near the cathode. The key here is the enhancement of the electric field near the cathode in the prebreakdown phase. If the externally applied field evolved much more slowly and all ions reached the dielectric surface of the cathode, a full shielding of the electric field over the entire gap would take place and no strong field could be concentrated anywhere in the gap space.

We thank Professor Uwe Czarnetzki (Ruhr-University Bochum) for his helpful comments. This research was performed at the FRBGYR, with funding through the program Promotion of Environmental Improvement to
Enhance Young Researchers' Independence, the special coordination funds for promoting science and technology, Japan Ministry of Education, Culture, Sports, Science and Technology (MEXT). This research has been also funded in part by Grants-in-Aid for Scientific Research from Japan Society for the Promotion of Science/MEXT as well as AOARD (AOARD-10-4074).

*To whom all correspondence should be addressed. tsuyohito@ppl.eng.osaka-u.ac.jp

[1] C. Nessim, M. Boulos, and U. Kogelschatz, Eur. Phys. J. Appl. Phys. 47, 22819 (2009).

[2] P. Baroch, N. Saito, and O. Takai, J. Phys. D 41, 085207 (2008).

[3] G. Fridman, G. Friedman, A. Gutsol, A. B. Shekhter, V. N. Vasilets, and A. Fridman, Plasma Process. Polym. 5, 503 (2008).

[4] M. G. Kong et al., New J. Phys. 11, 115012 (2009).

[5] T. N. Jukes and K.-S. Choi, Phys. Rev. Lett. 102, 254501 (2009).

[6] Y.P. Raizer Gas Discharge Physics (Springer-Verlag, Berlin, 1991).

[7] E. Wagenaars, M.D. Bowden, and G. M. W. Kroesen, Phys. Rev. Lett. 98, 075002 (2007).

[8] T. Hoder, R. Brandenburg, R. Basner, K.-D. Weltmann, K. V. Kozlov, and H.-E. Wagner, J. Phys. D 43, 124009 (2010).

[9] Y. V. Yurgelenas and H.-E. Wagner, J. Phys. D 39, 4031 (2006).

[10] T. Ito, K. Kobayashi, U. Czarnetzki, and S. Hamaguchi, J. Phys. D 43, 062001 (2010).

[11] O. A. Evsin, E. B. Kupryanova, V. N. Ochkin, S. Y. Savinov, and S. N. Tskhai, Quantum Electron. 25, 278 (1995).

[12] D. A. Akimov et al., JETP Lett. 70, 375 (1999).

[13] S. N. Tskhai et al., J. Raman Spectrosc. 32, 177 (2001).

[14] T. Ito, K. Kobayashi, S. Mueller, D. Luggenhölscher, U. Czarnetzki, and S. Hamaguchi, J. Phys. D 42, 092003 (2009).

[15] S.C. Brown Basic Data of Plasma Physics (AIP, New York, 1993). 